\title{
Accuracy of Free Hand Dental Implant Placement in a Private Dental College Setup - A Radiographic Analysis
}

\author{
Sam Jebaraj ${ }^{1}$, Subhasree R. ${ }^{2}$, Thiyaneswaran N. ${ }^{3}$, Dimple Rani ${ }^{4}$ \\ 1, 2, 3, 4 Department of Implantology, Saveetha Institute of Medical and Technical Sciences, \\ Chennai, Tamilnadu, India.
}

\section{ABSTRACT}

\section{BACKGROUND}

The factors that influence the implant accuracy are the anatomy of the patient, the experience of the practitioner and the surgical approach. However, the relative importance of each factor is poorly understood. Favourable function, occlusion, aesthetics and implant loading patterns can be obtained by optimal positioning. The experience of the surgeon is of utmost importance for the skill of placing a dental implant with proper angulation. The purpose of this retrospective study was to determine the increased accuracy obtained in performing freehand surgical placement of implants.

\section{METHODS}

This study was conducted in the Department of Implantology. A total of 1216 postoperative periapical radiographs from which single implant placement with adjacent straight root morphology was collected from the department in the period of June 2019 to June 2020 and checked for angulation, practitioner's experience and the site of placement. Mesio-distal angulation was calculated. Statistical analysis was performed using the SPSS method.

\section{RESULTS}

The results showed accuracy in angulation and positioning of implants increased with an increase in experience of the dental surgeon (oral surgeons, prosthodontists, periodontists). Within the limitations of the study, it showed that the freehand dental implant placement angulation was less than a degree for experienced surgeons. The postgraduates had a better understanding and orientation of the angulation than the undergraduates. Moreover, the difficult site was the second and third quadrant to assess the angulation properly.

\section{CONCLUSIONS}

With proper assessment of the anatomy and with experience freehand dental implant placement shows significant results with less angulation which can be prosthetically accepted.

\section{KEY WORDS}

Free Hand Surgery, Guided Surgery, Angulation Of Implants.
Corresponding Author: Dr. Subhasree R., Senior Lecturer, Department of Prosthodontics, Saveetha Dental College and Hospitals, Saveetha Institute of Medical and Technical Sciences, Saveetha University, Chennai-77, Tamilnadu, India. E-mail:drsubhashree27@gmail.com

DOI: $10.14260 / j e m d s / 2021 / 707$

How to Cite This Article: Jebaraj S, Subhasree R, Thiyaneswaran N, et al. Accuracy of free hand dental implant placement in a private dental college setup - a radiographic analysis. J Evolution Med Dent Sci 2021;10(39):3492-3495, DOI: 10.14260/jemds/2021/707

Submission 09-11-2020,

Peer Review 09-09-2021,

Acceptance 16-09-2021,

Published 27-09-2021.

Copyright (C) 2021 Sam Jebaraj et al. This is an open access article distributed under Creative Commons Attribution License [Attribution 4.0 International (CC BY 4.0)] 


\section{BACKGROUND}

Almost all of the commercially available dental implants are with fairly good longevity and clinical success rate therefore the focus of research on dental implants has shifted to accurate angulation and positioning as this is also important to attain optimal prosthetic out-come. If the angulation and positioning of the implant are improper it automatically increases the chances of complications, for example, perforation created on a lingual plate of the inferior alveolar canal. Likewise, the prosthesis would be in a compromised state which leads traumatic occlusal forces to be transmitted onto the implants and also aesthetics of the prosthesis turns out to be poor.1,2,3 Proper implant positioning is required to ensure that a prosthesis design is adaptable with long-term maintenance and provides access for sufficient oral hygiene. . $^{4,5}$

The important factors for long-term implant success are proper planning of the ideal implant position and bringing out the accurate transfer of planned implant position to the surgical site. Canullo and his colleagues calculated that almost half of the peri-implantitis cases identified were traced back to the main cause which was improper positioning of the implant. ${ }^{\text {The }}$ actual outcome of conventional planning has been acquired with the use of a radiographic stent with the help of a radiopaque marker, obtained from building a wax-up of the actual prostheses on study models and radiographic stent worn by the patient while taking the pre-operative cone-beam computed tomography (CBCT) scan, thus paving a way for transposition of the ideal prosthesis shape to the alveolar ridge and defining the ideal prosthetic position for the implant to be placed. The radiographic stent can thereafter be converted into a surgical stent, thus helping the surgeon to obtain a view of the ideal prosthetic position intraoperatively.

Various techniques for surgical guidance have shown to be effective in increasing the accuracy of implant placement. ${ }^{8}$ But still, guided surgery could not be the choice of method always because of lack of resources or the emergency status of the case. ${ }^{9-11}$ On the other hand with appropriate presurgical planning, which includes 3-dimensional radiographic imaging12,13,14 and also proper case selection, freehand surgery will be an acceptable alternative method. Thus, it is critical to find out the factors which affect the accurate positioning of the implant fixture.

Generally, the surgeon makes a decision in situ on the chosen implant position after the flap is raised and the bone is being exposed. 15 The drawback of this method is that the final angulation, depth and position of the implant to be placed are finalized by the surgeon intraoperatively. Possible surgical stents are just used as a supplement to visualize the prosthetic position. Hence, this method is often called to be "freehand", and the accuracy of the final implant position is directly proportional to the surgeon's skill and experience. Previous researches conducted regarding factors influencing implant positioning in free-hand surgery significantly were focused on surgical drilling methods and the surgeon's years of experience. Also, these studies gave certain important information regarding the effect of drilling speed, making use of all bur diameters in succession, and also inter-observer differences. ${ }^{16}$ Because these factors affecting implant accuracy in freehand cases have not been systematically investigated yet, surgeons are the ones to make decisions about choosing guided or freehand surgery largely based on their preference or experience. The intention of this retrospective study was to prove that the accuracy of freehand surgery improved as the experience of the surgeon increased and thereby guided surgery could be avoided in a lot of single implant cases.

\section{METHODS}

This retrospective study was conducted in the Department of Implantology in Saveetha Dental College and Hospitals, Saveetha Institute of Medical and Technical Sciences, Chennai from March 2019 to March 2021. A total of 1216 postoperative periapical radiographs were collected from the department with certain inclusion criteria - single tooth missing cases, straight root morphology and exclusion criteria as multiple missing teeth, curved roots. Apart from these all the other cases were included in the study. Patients belonging to the age group ranging from 20 - 60 years were included in the study. Auto cad was used for angle determination. Mesiodistal angulation of the implant placed was calculated. Cases for which inter-observer values were taken into consideration while placing implants were included in the study. Amongst the 1216 radiographs collected, 705 freehanded surgery postoperative periapical radiographs were included.

Postoperative intraoral periapical radiographs were taken and measured. A1 and A2 were two determinants. (Figure 1) A1 represents the angle of the implant placed and A2 represents the angle of the adjacent tooth. A1-A2 determines the angle differentiation of the tooth and the implant. The obtained angulation difference is then associated with factors such as the site of the implant, educational qualification of the student placing the implant and experience of the guide.

\section{Statistical Analysis}

The collected radiographs were analyzed and tabulated using excel sheets followed by chi-square test statistical analysis using the SPSS software.

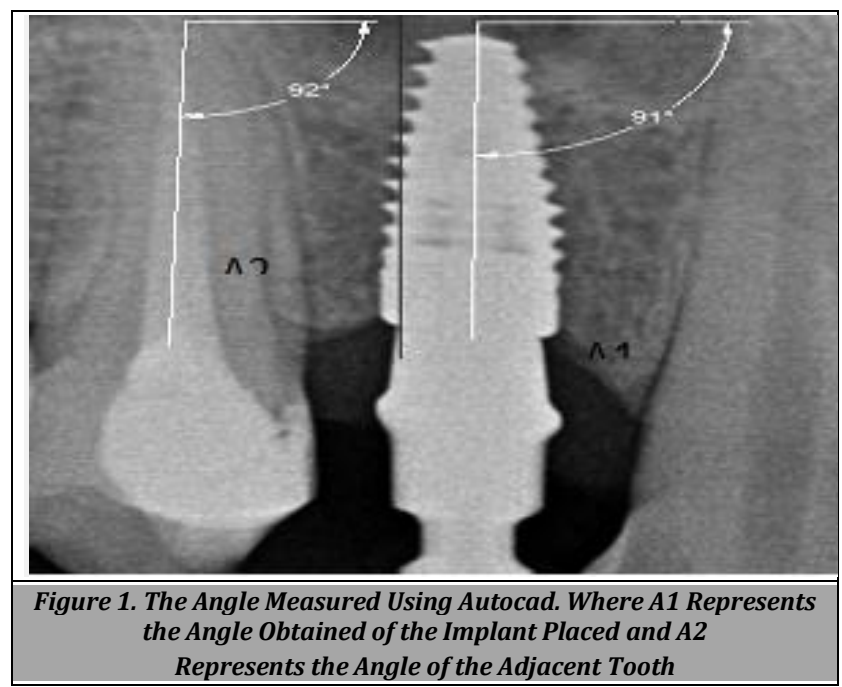




\section{RESULTS}

The association between the angulation and the experience of the guide revealed that $9.3 \%, 37.1 \%, 53.6 \%$ of $<5$ years, 5 10 years, $>10$ years of experience were among 0 degree angulation. $27.3 \%, 41.1 \%, 31.6 \%$ of $<5$ years, $5-10$ years, > 10 years of experience were among \pm 1 degree angulation. $43.1 \%, 35.8 \%, 21.2 \%$ of $<5$ years, $5-10$ years, $>10$ years of experience were among \pm 2 degree angulation. $56.7 \%, 28.4$ $\%, 14.9 \%$ of $<5$ years, 5-10 years, $>10$ years of experience were among \pm 3 degree angulation. Chi-square association was done and found to be statistically significant. Chi-square value: 72.197 , df: 6 , P-value: $0.00<0.05$. (Figure 2) The association between the angulation and the educational qualification of the student revealed that $26.8 \%, 73.2 \%$ of UG and PG respectively were among 0 degree angulation, $63.2 \%, 36.8 \%$ of UG and PG respectively were among \pm 1 degree angulation, $62.5 \%, 37.5 \%$ of UG and PG respectively were among \pm 2 degree angulation, $80.6 \%, 90.4 \%$ of UG and $\mathrm{PG}$ respectively were among \pm 3 degree angulation. Chisquare association was done and found to be statistically significant. Chi-square value: 57.984, df: 3, P-value: $0.00<$ 0.05 . (Figure 3 ) The association between the angulation and the sextant of the tooth revealed that $15.5 . \%, 19.6 \%, 13.4 \%$, 14.4. \%, $23.7 \%, 13.4 \%$ of 1-6 sextants respectively were among 0 degree angulation, $10.3 \%, 9.5 \%, 11.5 \%, 26.1 \%$, $10.7 \%, 32.0 \%$ of $1-6$ sextants respectively were among \pm 1 degree angulation, $13.2 \%, 13.5 \%, 11.1 \%, 24.3 \%, 5.9 \%$, $31.9 \%$ of $1-6$ sextants respectively were among \pm 2 degree angulation, $9.0 \%, 9.0 \%, 9.0 \%, 31.3 \%, 9.0 \%, 32.8 \%$ of $1-6$ sextants respectively were among \pm 3 degree angulation. Chisquare association was done and found to be statistically significant. Chi-square value: 47.699 , df: 15 , P-value: $0.00<$ 0.05. (Figure 4).

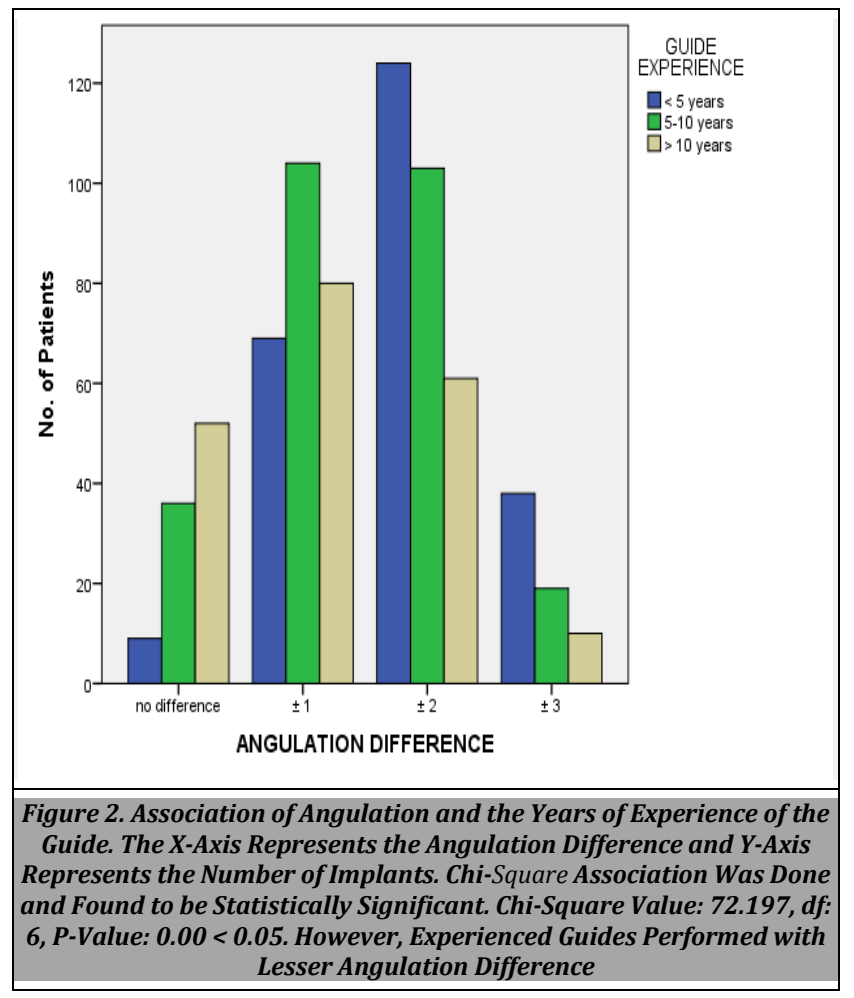

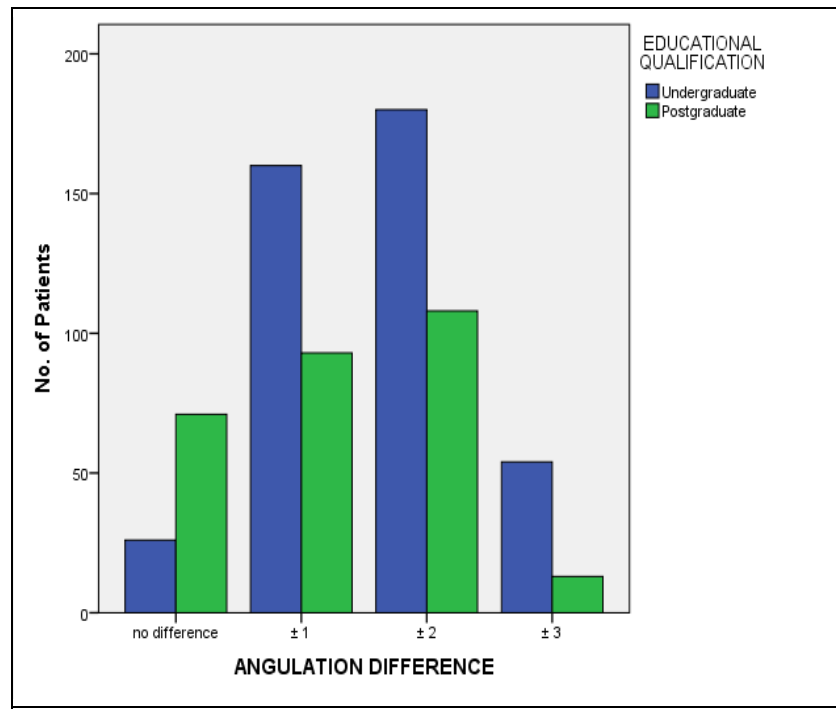

Figure 3. Association of Angulation and the Educational Qualification of the Student. The X-Axis Represents the Angulation Difference and $Y$ Axis Represents the Number of Implants. Chi-Square Association Was Done and Found to be Statistically Significant. Chi-Square Value: 57.984, df: 3, P-Value: $0.00<0.05$. However, Postgraduates Performed with Lesser Angulation Difference

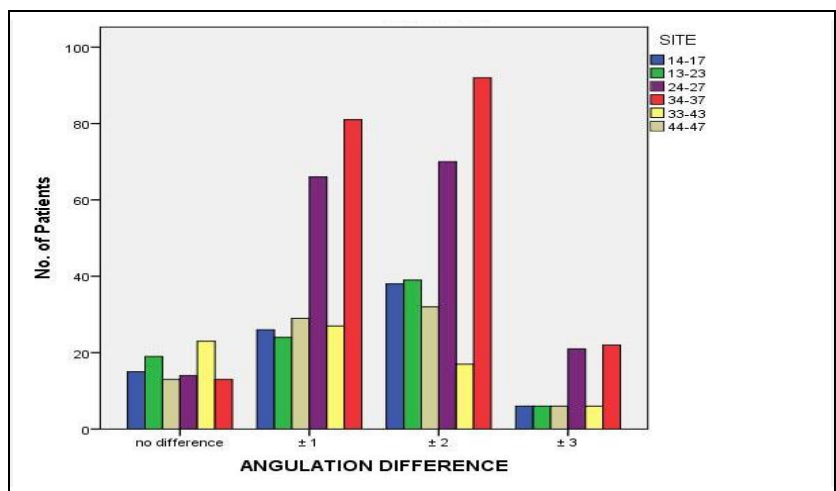

Figure 4. Association of Angulation and the Sextant of the Tooth. X-Axis Represents the Angulation Difference and Y-Axis Represents the Number of Implants. Chi-Square Association Was Done and Found to be Statistically Significant. Chi-Square Value: 47.699, df: 15, P-Value: $0.00<0.05$. However, Anterior Sextants Show Lesser Angle Difference

\section{DISCUSSION}

This study included 3 factors, site of implant, educational qualification and guide experience to influence the accuracy of implant placement. Adjacent teeth were measured along with implants.

Smith et al. suggested that tooth replacement utilizing dental implants is a major clinical challenge in anatomically or aesthetically demanding regions and correct implant position, angulation and insertion depth determine functional and aesthetic success (Smith \& Zarb 1989; Stanford 1999; Andersen et al. 2002; Lorenzoni et al. 2003; Palmer et al. 2003; Kramer et al. 2005).

Koop et al. concluded that even though CT- supported insertion guides might be more accurate and provide an improved three-dimensional representation of the surgical area, they involve specific equipment being not available in most dental offices and thus entail additional costs to the patient (Kopp et al. 2003; Windhorn 2004). 
Tarnow et al. stated that, in the aesthetic zone, precise mesiodistal implant placement is a prerequisite for maintenance of the peri-implant bone. According to Tarnow et al. $(2000,2003)$, a minimum distance of $3 \mathrm{~mm}$ between two implants and a minimum distance of $1.5 \mathrm{~mm}$ between tooth and implant are elementary to maintain proper soft tissue contours despite a physiologic remodelling of the peri implant tissues.

Michael Payer et al. concluded that, in order to achieve precise implant angulation, all bur diameters available should be used. Utilizing low drilling speeds results in less mesiodistal deviation. The surgeon's experience seems to be the most relevant factor in precise implant placement.

The practitioners' experience plays a major role in factors contributing to implant angulation. Single implants are ideal for free-hand surgery, and for multiple implants special care is needed. The possibility of approaching the root of an adjacent tooth should be considered in free hand surgery. From the data obtained postgraduates have better positional accuracy when compared to undergraduates. The experience of staff who guided students, the more the experience larger the accuracy of implants.

Single tooth implant placement and immediate implant placement cases performed with the help of experienced surgeons proved to be more accurate in terms of angulation and positioning whereas guided surgery would be appropriate for cases with multiple implant placements.

\section{CONCLUSIONS}

Guided surgery is becoming famous for accurate implant placement, however not every patient is affordable. Within the limitations of this study, it showed that the free hand dental implant placement angulation was less than a degree for experienced surgeons. The postgraduates had better understanding and orientation of the angulation than the undergraduates. Moreover, the difficult sites were the second and third quadrants to assess the angulation properly. With proper assessment of the anatomy and with experience free hand dental implant placement shows significant results with less angulation which can be prosthetically accepted.

Data sharing statement provided by the authors is available with the full text of this article at jemds.com.

Financial or other competing interests: None.

Disclosure forms provided by the authors are available with the full text of this article at jemds.com.

\section{REFERENCES}

[1] De Angelis F, Papi P, Mencio F, et al. Implant survival and success rates in patients with risk factors: results from a long-term retrospective study with a 10 to 18 years follow-up. Eur Rev Med Pharmacol Sci 2017;21(3):4337.

[2] Derks J, Tomasi C, Wennström Jl, et al. Response to letter to the editor. Effectiveness of implant therapy analyzed in a Swedish population: early and late implant loss. Journal of Dental Research 2015;94(Suppl 3):44S-51S.
[3] van Velzen Fjj, Ofec R, Schulten EAJM. 10-Year survival rate and the incidence of peri-implant disease of 374 titanium dental implants with a sla surface: a prospective cohort study in 177 fully and partially edentulous patients. Clin Oral Implants Res 2015;26(10):1121-8.

[4] Van Assche N, Vercruyssen M, Coucke W, et al. Accuracy of computer-aided implant placement. Clinical Oral Implants Research 2012;23(Suppl 6):112-23.

[5] Cooper LF. Prosthodontic complications related to nonoptimal dental implant placement. Dental Implant Complications 2015;2:539-58.

[6] Tahmaseb A, Wismeijer D, Coucke W, et al. Computer technology applications in surgical implant dentistry: a systematic review. Int J Oral Maxillofac Implants 2014;(Suppl 29):25-42.

[7] Canullo L, Tallarico M, Radovanovic S, et al. Distinguishing predictive profiles for patient-based risk assessment and diagnostics of plaque induced, surgically and prosthetically triggered peri-implantitis. Clin Oral Implants Res 2016;27(10):1243-50.

[8] Akça K, Iplikçioğlu H, Cehreli MC. A surgical guide for accurate mesiodistal paralleling of implants in the posterior edentulous mandible. J Prosthet Dent 2002;87(2):233-5.

[9] Besimo C, Lambrecht JT, Nidecker A. Dental implant treatment planning with reformatted computed tomography. Dentomaxillofacial Radiology 1995;24(4):264-7.

[10] Ersoy AE, Turkyilmaz I, Ozan O, et al. Reliability of implant placement with stereolithographic surgical guides generated from computed tomography: clinical data from 94 implants. J Periodontol 2008;79(8):133945.

[11] Fortin T, Bosson JL, Coudert JL, et al. Reliability of preoperative planning of an image-guided system for oral implant placement based on 3-dimensional images: an in vivo study. The Int J Pral Maxillofac Implants 2003;18(6):886-93.

[12] BouSerhal C, Jacobs R, Quirynen M, et al. Imaging technique selection for the preoperative planning of oral implants: a review of the literature. Clin Implant Dent Relat Res 2002;4(3):156-72.

[13] Lam EWN, Ruprecht A, Yang J. Comparison of twodimensional orthoradially reformatted computed tomography and panoramic radiography for dental implant treatment planning. The Journal of Prosthetic Dentistry 1995;74(1):42-6.

[14] Benavides E, Rios HF, Ganz SD, et al. Use of cone beam computed tomography in implant dentistry: the International Congress of Oral Implantologists consensus report. Implant Dent 2012;21(2):78-86.

[15] Hoffmann J, Westendorff C, Gomez-Roman G, et al. Accuracy of navigation-guided socket drilling before implant installation compared to the conventional freehand method in a synthetic edentulous lower jaw model. Clinical Oral Implants Research 2005;16(5):609-14.

[16] Payer M, Kirmeier R, Jakse N, et al. Surgical factors influencing mesiodistal implant angulation. Clin Oral Implants Res 2008;19(3):265-70. 